\title{
Multivariable analysis of host amino acids in plasma and liver during infection of malaria parasite Plasmodium yoelii
}

\author{
Erisha Saiki ${ }^{1,2}$, Kenji Nagao ${ }^{3}$, Hiroka Aonuma ${ }^{1}$, Shinya Fukumoto ${ }^{2}$, Xuenan Xuan², Makoto Bannai ${ }^{3}$ \\ and Hirotaka Kanuka ${ }^{1,2^{*}}$
}

\begin{abstract}
Background: Malaria is the most significant human parasitic disease, and yet understanding of the energy metabolism of the principle pathogen, Plasmodium falciparum, remains to be fully elucidated. Amino acids were shown to be essential nutritional requirements since early times and much of the current knowledge of Plasmodium energy metabolism is based on early biochemical work, performed using basic analytical techniques, carried out almost exclusively on human plasma with considerable inter-individual variability.

Methods: In order to further characterize the fate of amino acid metabolism in malaria parasite, multivariate analysis using statistical modelling of amino acid concentrations (aminogram) of plasma and liver were determined in host infected with rodent malaria parasite, Plasmodium yoelii.

Results and conclusion: Comprehensive and statistical aminogram analysis revealed that $P$. yoelii infection caused drastic change of plasma and liver aminogram, and altered intra- and inter-correlation of amino acid concentration in plasma and liver. These findings of the interactions between amino acids and Plasmodium infection may provide insight to reveal the interaction between nutrients and parasites.
\end{abstract}

Keywords: Plasmodium yoelii, Infection, Nutrition, Amino acid, Aminogram, Multivariable analysis

\section{Background}

Malaria, the most significant human parasitic disease, remains a major cause of morbidity, anaemia, and mortality, in particular in developing countries. Malaria currently accounts for about two to three million deaths each year and estimates have been increasing over the last three decades [1]. It has long been acknowledged that populations residing in malaria-endemic areas generally live under conditions that lead to poor nutritional status. The groups at highest risk of the adverse effects of malaria, children and pregnant women, are also most affected by malnutrition. Infection by the human malaria parasite Plasmodium falciparum has severe and potentially lethal

\footnotetext{
* Correspondence: kanuka@jikei.ac.jp

${ }^{1}$ Department of Tropical Medicine, The Jikei University School of Medicine, 3-25-8, Nishi-Shinbashi, Minato-ku, Tokyo 105-8461, Japan

${ }^{2}$ National Research Center for Protozoan Diseases, Obihiro University of Agriculture and Veterinary Medicine, Inada-cho, Obihiro, Hokkaido 080-8555, Japan

Full list of author information is available at the end of the article
}

consequences for the metabolic state of the human host [2]. In all parasitic infections, there is a significant metabolic interaction between pathogen and host as the parasite diverts nutrients towards its own growth while the host exerts to maintain homeostasis. In the case of $P$. falciparum infection, the huge metabolic demands of the rapidly proliferating parasite cells, coupled with the effects of massive erythrocyte lysis, are responsible for the pathogenesis of the disease and its clinical manifestations, which include hypoglycaemia, lactic acidosis, haemolytic anaemia, haemoglobinuria and hypoargininaemia.

Despite the clinical and economic significance of $P$. falciparum, the energy metabolism of this organism is still poorly understood. Amino acids were shown to be essential nutritional requirements of the parasite a century ago [3]. For example, the severity of malaria is related to arginine concentration in plasma and L-arginine administration to cerebral malaria patients reverts endothelial dysfunction $[4,5]$. Extensive genomic and biochemical

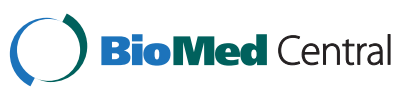


evidence indicate that many parts of parasite metabolism have been radically rationalized or modified. Plasmodium parasites are incapable of amino acid biosynthesis, relying on haemoglobin catabolism and uptake from the extracellular space [6]. Upon invading an erythrocyte, the parasite initiates a catabolic process whereby haemoglobin from the erythrocyte cytoplasm is ingested and proteolysed into its constituent amino acids in an acidic vacuole [7]. Therefore, the growth and multiplication of parasites are dependent on the presence in the extracellular solution of a set of amino acids, for example, isoleucine or methionine [8-11], that are absent or very little from adult human haemoglobin [12]. In addition, amino acids are substrate of proteins and play a central role as intermediates in nutrition metabolism, which form complicated networks, such as lipid, nitrogen and carbohydrate [13]. Thus, a novel analytic method is required to be developed in a blanket manner to understand the interaction among parasites and host amino acid metabolisms.

This study aimed to perform multivariable analysis using statistical modelling of amino acid concentrations (aminogram) [14,15] of plasma and liver of the host that was infected by rodent malaria parasite, Plasmodium yoelii. Plasma aminogram analysis indicates comprehensive survey of the amino acid concentrations in plasma, which has already been used in diagnosis of cancer [16]. Due to the importance of Plasmodium amino acid metabolism for malaria pathogenesis and as a target for most current candidate anti-malarial pharmaceuticals, it is critical to understand the dynamics of the amino acid metabolic networks in Plasmodium subspecies. Alternative approaches to reconstructing these networks, such as presumption from genomic data or in vitro biochemistry, are currently incomplete and metabolomics technologies are beginning to enable systems-level measurements of changes in metabolic activity $[17,18]$. This study provides a novel view to reveal the host-parasite interaction in aspects of nutrition dynamics, and the role played by these complicated pathways in maintaining the host-parasite balance.

\section{Methods}

\section{Ethics statement}

This study was carried out in strict accordance with the recommendations in the Guide for the Laboratory Animals of the Obihiro University of Agriculture and Veterinary Medicine and The Jikei University School of Medicine. The protocol was approved by the Committee on the Animal Experiments of the Obihiro University of Agriculture and Veterinary Medicine (Permit Number: 21-41 and 21-42) and The Jikei University School of Medicine (Permit Number: 23-020). All experiments using mice were performed under anaesthesia, and all efforts were made to minimize suffering in accordance with the Guidelines for Animal Experimentation of the
Japanese Association for Laboratory Animal Science and the Fundamental Guidelines for Proper Conduct of Animal Experiment and Related Activities in Academic Research Institutions under the jurisdiction of the Ministry of Education, Culture, Sports, Science and Technology, Japan.

\section{Animals}

Six- to eight-week-old male BALB/c mice (CLEA Japan) were infected with the $P$. yoelii $17 \mathrm{XL}$ strain by the intraperitoneal injection of infected blood. Asexual growth was monitored by Giemsa-stained smear. The animal room was maintained at a constant temperature $\left(23 \pm 2^{\circ} \mathrm{C}\right)$ and humidity $(55 \pm 10 \%)$ with a $12: 12$ hours light-dark cycle (lights on 0800-2000). Under diethylether anaesthesia, blood and liver were collected. The liver samples were immediately frozen and stored at $-80^{\circ} \mathrm{C}$ until analysis.

\section{Parasite infection}

Donor BALB/c mice were intraperitoneally injected with $1 \times 10^{6}$ erythrocytes infected with $P$. yoelii $17 \mathrm{XL}$ strain $[19,20]$. Parasitaemia was monitored by counting the number of parasite-infected erythrocytes per 1,000 erythrocytes by microscopic examination of Giemsa-stained, thin (tail) blood smears.

\section{Amino acid analysis}

Animals were bled under ether anesthesia; blood was collected into tubes containing EDTA as anticoagulant. After centrifugation, the supernatant was used for examination of plasma. Plasma samples were mixed with two volumes of $5 \%(\mathrm{w} / \mathrm{w})$ trichloroacetic acid, and centrifuged $\left(4^{\circ} \mathrm{C}, 20 \mathrm{~min}, 8,000 \mathrm{~g}\right)$ immediately after blood collection to remove precipitated protein. To prepare deproteinized tissue extracts, tissues were homogenized by using $5 \%$ trichloroacetic acid as described previously [21]. All samples were kept at $4^{\circ} \mathrm{C}$ during all steps to minimize chemical reactions of thiol metabolites. The amino acid concentrations were measured by an automatic amino acid analyzer (L-8800; Hitachi, Tokyo, Japan). Briefly, amino acids, separated by cation exchange chromatography, were spectrophotometrically detected after post-column reaction with ninhydrin reagent. Hydroxyproline content was determined by the method previously reported [22]. 20 amino acids (Asn, Gln, Asp, Thr, Ser, Glu, Pro, Gly, Ala, Cys, Val, Met, Ile, Leu, Tyr, Phe, His,, Lys, Trp, Arg), 11 their derivative (Hydroxyproline (Hypro), Hydroxylysine (Hylys), 3-methylhistidine (3-MeHis), 1-methylhistidine (1-MeHis), ethanolamine $\left(\mathrm{EOHNH}_{2}\right)$, beta-alanine (beta-Ala), alpha-aminobutyric acid (alpha-ABA), Phosphoethanolamine (PEA), Taurine (Tau), Ornithine (Orn), Citrullline (Cit)), urea, and ammonia $\left(\mathrm{NH}_{3}\right)$ were measured in this study. 

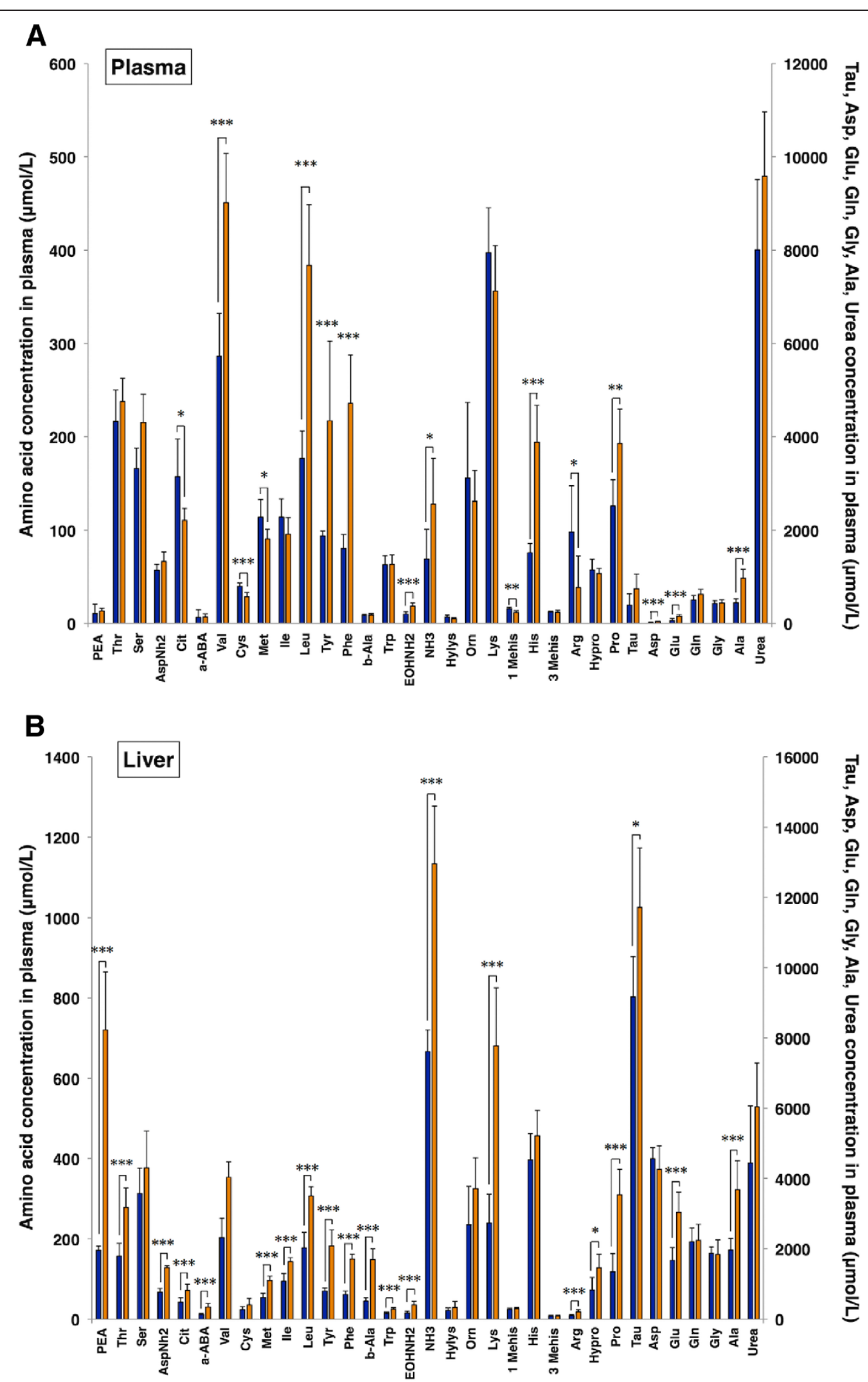

Figure 1 (See legend on next page.) 
(See figure on previous page.)

Figure 1 Plasma and liver amino acid concentrations vary in host infected with Plasmodium yoelii. Amino acid concentrations in plasma (A) and liver (B) were analysed on five days post-infection with Plasmodium yoelii. Shown are amino acids concentrations of uninfected mice (blue bars), infected mice (orange bars). Each bar represents mean \pm SD of six mice. Asterisks denote: ${ }^{*} p<0.05 ;{ }^{* *} p<0.01 ;{ }^{* * *} p<0.001$. BALB/c mice were infected with $1 \times 10^{6} \mathrm{RBC}$ parasitized with $P$. yoelii.

\section{Aminogram analysis}

Aminogram analysis was performed as previously described [14]. All data of plasma and liver concentrations of amino acids were analysed by using $\mathrm{R}$ (version 2.12.1; $\mathrm{R}$ foundation for statistical computing, Vienna, Austria [23]) for hierarchical cluster analysis. $R$ was also used to construct coloured blocks in aminogram representing levels of amino acid concentrations, and to draw dendrogram using Ward's method [14]. The value for each amino acid concentration was normalized to a z score by using the mean and standard deviations of the scores.

\section{Spearman's rank correlation coefficient analysis}

Intra- or inter-correlations among amino acid concentrations in plasma and liver were tested by non-parametric equivalent Spearman's rank correlation co-efficient analysis $[24,25]$. The calculations were performed with $\mathrm{R}$, and

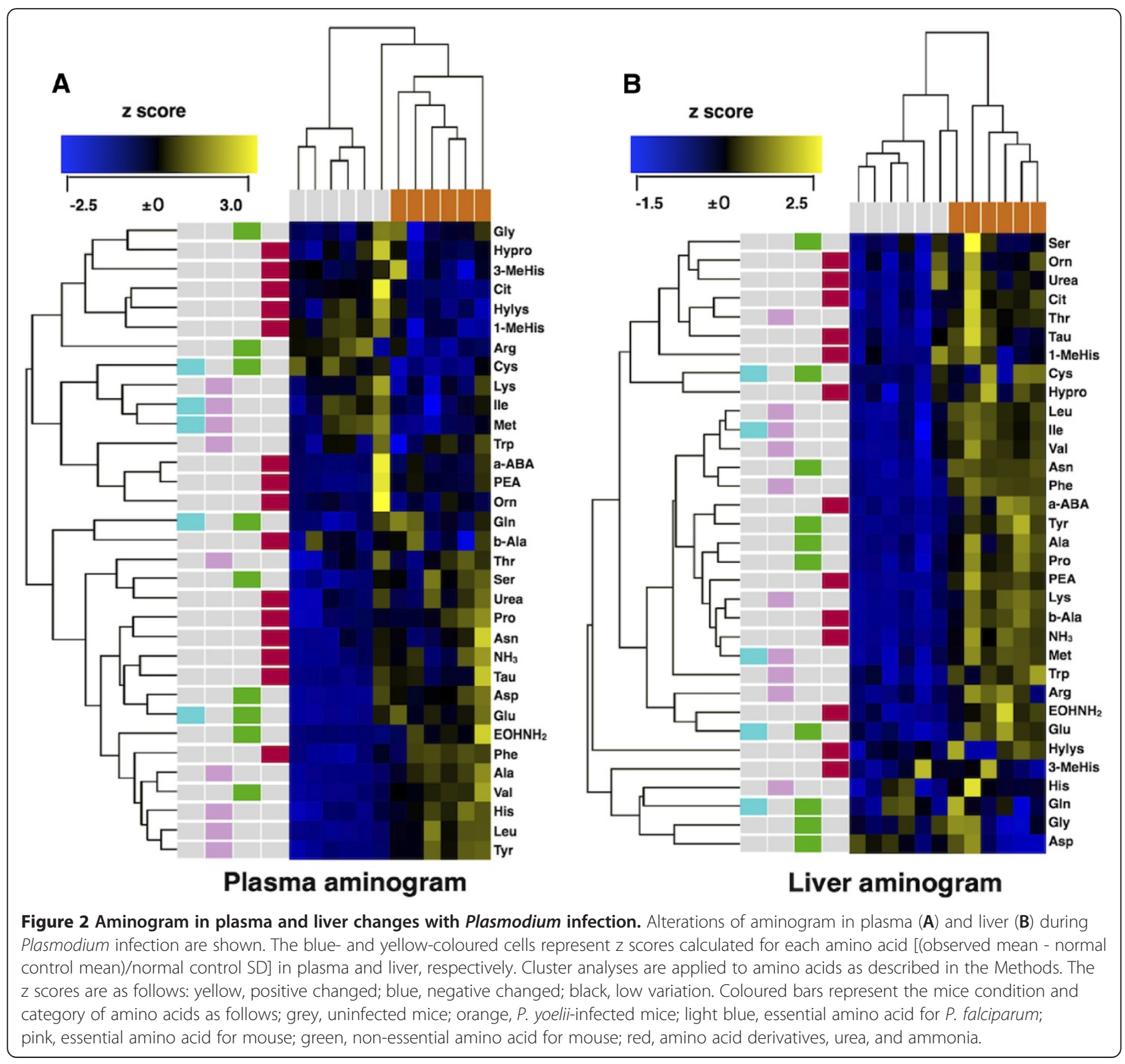


the results of pair-wise correlations are shown as heat map $(|\rho| \geqq 0.7, p<0.05)$.

\section{Results}

Plasma and liver amino acid concentrations vary in host infected with Plasmodium yoelii

To investigate amino acid metabolic profile in host during Plasmodium infection, concentrations of 20 amino acids, 11 their derivative, urea, and ammonia in plasma and liver were analysed in $P$. yoelii-infected mice whose parasitaemia was $84.5 \pm 3.3 \%$. Ten plasma amino acids (Val, Leu, Tyr, Phe, EOHNH 2 , His, Pro, Asp, Glu, Ala) and $\mathrm{NH}_{3}$ significantly increased, and five amino acids (Cit, Cys, Met, 1-MeHis, Arg) significantly decreased (Figure 1A). On the other hand, 21 liver amino acids (PEA, Thr, Asn, Cit, $\alpha$-ABA, Met, Ile, Leu, Tyr, Phe, $\beta$-Ala,
Trp, EOHNH ${ }_{2}$, Lys, Arg, HyPro, Pro, Tau, Glu, Ala) and $\mathrm{NH}_{3}$ significantly increased, and no amino acids significantly decreased (Figure 1B). Plasma and liver aminogram analysis based on heat-map [26] and cluster analysis [27] was then performed, demonstrating that individual plasma and liver aminogram were drastically altered by $P$. yoelii infection as well as forming infection-dependent cluster (Figures 2A and B). These data suggested that Plasmodium infection causes drastic alterations in host amino acid metabolism, potentially in a common pattern specific and characteristic of Plasmodium infection.

Intracorrelation of aminogram variation in each tissue To better understand the relationship between Plasmodium parasites and host amino acid levels, multivariate-

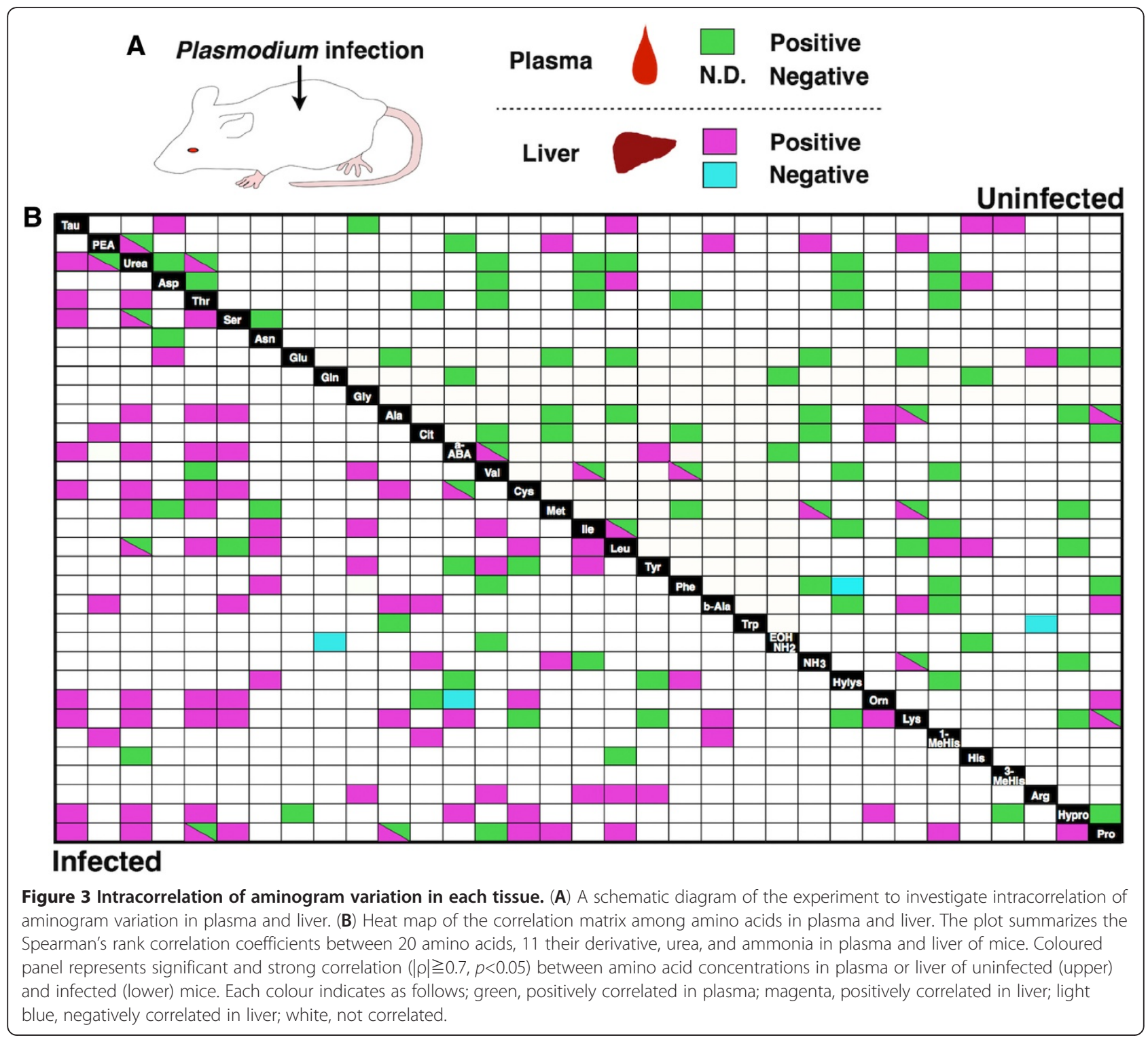


correlation analysis of amino acid concentrations in each of plasma and liver was performed. Spearman's rank correlation co-efficient analysis was applied to plasma and liver samples from mice infected with $P$. yoelii. The scatterplots of 2 plasma amino acids show various patterns, such as positive and negative linear correlations or no correlations (data not shown). The results were summarized as heat-map based on Spearman's rho and $p$-value (Figures $3 \mathrm{~A}$ and $\mathrm{B}$ ). In uninfected mice, the numbers of positively correlated pairs in amino acid concentrations were $67 / 528$ (12.6\%) in plasma and $31 / 528$ (5.87\%) in liver. In infected mice, the number of positively correlated pairs in plasma decreased to $28 / 528$ (5.30\%), while the number in liver increased to $81 / 528$ (15.3\%). Furthermore, Spearman's test between parasitaemia and alteration of amino acid concentrations showed positive (Asp, Gly, His) and negative correlation (Tyr) $(|\rho| \geqq 0.7$, $p<0.05)$. These results indicated that Plasmodium infection induced alterations in intra-correlations of amino acid concentrations in each tissue.

Inter-correlation of aminogram variation between plasma and liver

The amino acid transportations among organs are intermediated by plasma. In order to acquire further information about the external interaction between plasma and liver, Spearman's test for comparing correlations between plasma and liver amino acid concentrations was carried out. The number of positively correlated pairs in amino acid concentrations between plasma and liver were 16/ 1089 (1.47\%) in uninfected mice, and 16/1089 (1.47\%) in infected mice (Figures $4 \mathrm{~A}$ and B). No pairs of negatively correlated were determined in uninfected mice, while pairs in infected mice were 23/1089 (2.11\%). Interestingly, correlated pairs in uninfected mice did not match to any pairs in infected mice. These results indicated

A

\section{Plasmodium infection}

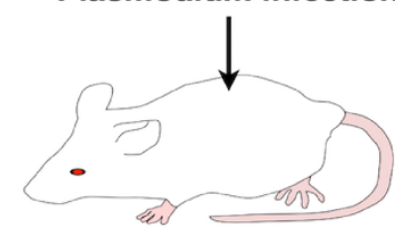

Plasma

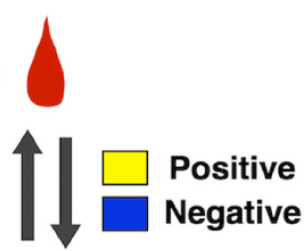

Liver

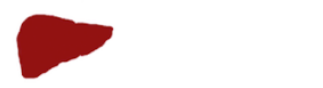

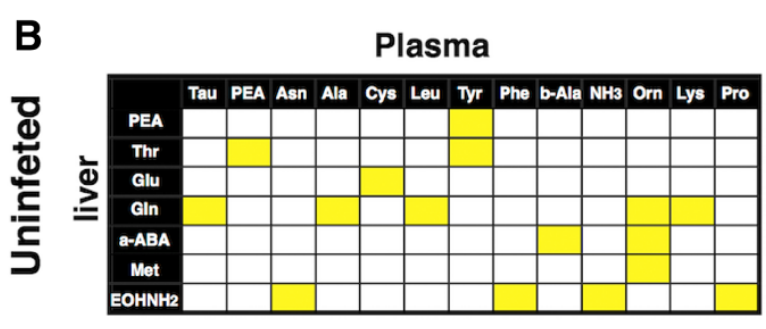

Plasma

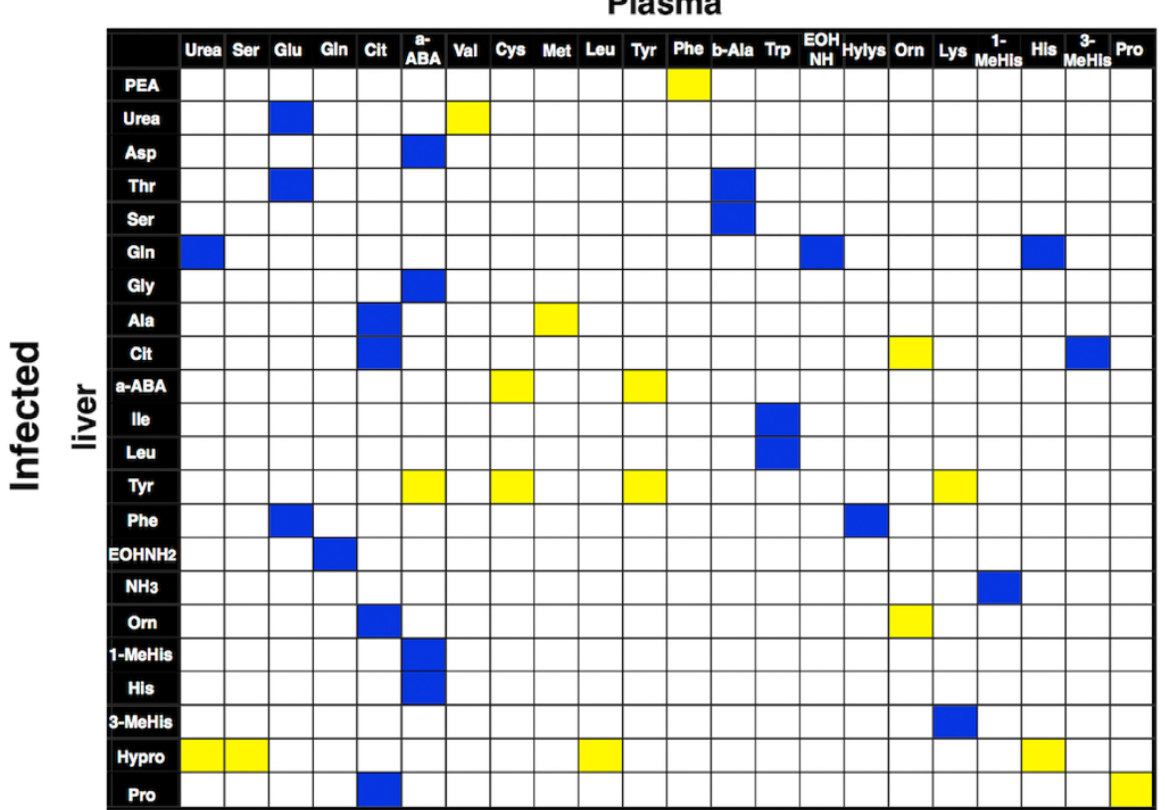

Figure 4 Intercorrelation of aminogram variation between plasma and liver. (A) A schematic diagram of the experiment to investigate intercorrelation of aminogram variation between plasma and liver. (B) Heat map of the correlation matrix among amino acids between plasma and liver. The plot summarizes the Spearman's rank correlation coefficients among amino acids and their derivatives between plasma and liver. Coloured panel represents significant and strong correlation $(|\rho| \geqq 0.7, p<0.05)$ between plasma and liver amino acid concentrations in uninfected (upper) and infected (lower) mice. Each colour indicates as follows; yellow, positively correlated; blue, negatively correlated; white, not correlated. 
that parasite infection drastically changed relationships between plasma and liver amino acid concentrations.

\section{Discussion}

Host-pathogen interactions in malaria rely on an exchange of nutrients, specifically amino acids, between erythrocyte and parasites. Amino acid concentrations of plasma were constantly kept in a set range to maintain amino acid homeostasis, although the results indicated that the concentrations are highly varied during Plasmodium infection (Figure 1A). The alterations of plasma amino acid concentrations by parasite infection are considered to be the results of export/import promotion of amino acids from/ to erythrocytes, erythrocyte rupture, immunological enhancement, and acceleration of haematopoiesis. Since the changes of amino acid concentrations in liver influenced on plasma amino acid concentrations (Figure 1B), the variations of amino acid concentrations in other organs may also alter them. Furthermore, the enormous complexity of nutrition network makes it difficult to maintain amino acid homeostasis during parasite infection. Therefore, aminogram may lose the constancy among individual hosts during Plasmodium infection, although plasma and liver aminogram kept the potentially homogeneous pattern (Figures 2A and B). Amino acids are transported through blood, and their metabolism plays a central role in nutrient metabolisms; in other words, plasma aminogram indirectly reflects the metabolic dynamics in the host [28]. Consequently, these findings suggested specific patterns of nutrition dynamics responding to Plasmodium infection.

Metabolomic analysis gives us deeper understanding of Plasmodium biology and malaria patient disease state. Aminogram analysis revealed the variational aspects of host amino acid concentrations during Plasmodium infection, and the results suggested that altered aminogram develop novel relationships between amino acids. Furthermore, the results indicated that Plasmodium infection changed the intercorrelation of amino acids in plasma as well as in liver. Since amino acids act as growth regulation factors for parasites, it is reasonable to hypothesize that there is suitable plasma aminogram for parasite proliferation. These findings suggested that Plasmodium infection disrupted the original link and established a new one between amino acids (Figures 4A and B); that is, infection leads to novel amino acid dynamics. Meanwhile, Plasmodium parasites convert property of erythrocyte into adequate condition for themselves by exporting many proteins to plasma [29], such as degraded haemoglobin [30]. In fact, positive correlation was shown between plasma aminogram and amino acid composition of haemoglobin (Spearman's $\rho=0.46, p<0.05$ ), proposing a new model that Plasmodium parasites adjust amino acid dynamics of host to an appropriate state for themselves.
In summary, this study demonstrated that aminogram analysis and multivariate-correlation analysis of plasma and liver amino acids were efficient, to reveal the complicated interaction between parasites and host metabolisms. Through these analyses, a view to renew the traditional concepts of interaction between parasites and host was obtained. The expansion from metagenome to metabolome [31], such as aminogram analysis, is a promising approach to characterize life phenomenon, to find new candidate anti-malarial pharmaceuticals or preventative measures, and to give fresh insights into malaria control.

\section{Competing interests}

The authors declare that they have no competing interests.

\section{Authors' contributions}

ES and HK conceived the study and wrote the paper. ES, KN, and MB carried out the aminogram analysis. ES, HA, SF and XX contributed to the data analysis and discussion. All the authors read and approved the final manuscript. MB and HK supervised the study.

\section{Acknowledgements}

We are grateful to Motomi Torii for the Plasmodium strain and other laboratory members for their enthusiastic participation. We are grateful to $\mathrm{K}$ Bandai and Y Doi for skilful technical assistance, and R Paudel and K Miura for valuable discussions. This study was supported in part by Grants-in-Aid for Scientific Research from the Japanese Ministry of Education, Science, Sports, Culture and Technology to HK and SF, the Ohyama Health Foundation to HK, and the Funding Program for Next Generation WorldLeading Researchers (NEXT Programme) to HK. HA is a research fellow of the Japan Society for the Promotion of Science.

\section{Author details}

'Department of Tropical Medicine, The Jikei University School of Medicine, 3-25-8, Nishi-Shinbashi, Minato-ku, Tokyo 105-8461, Japan. ${ }^{2}$ National Research Center for Protozoan Diseases, Obihiro University of Agriculture and Veterinary Medicine, Inada-cho, Obihiro, Hokkaido 080-8555, Japan. ${ }^{3}$ Institute for Innovation, Ajinomoto Co Inc, Kanagawa 210-8680, Japan.

Received: 8 December 2012 Accepted: 15 January 2013 Published: 16 January 2013

\section{References}

1. Krogstad DJ: Malaria as a reemerging disease. Epidemiol Rev 1996, 18:77-79.

2. Planche T, Dzeing A, Ngou-Milama E, Kombila M, Stacpoole PW: Metabolic complications of severe malaria. Curr Top Microbiol Immunol 2005, 295:105-136.

3. Barry DN: Metabolism of babesia parasites in vitro: amino acid production by Babesia rodhaini compared to Plasmodium berghei. Aust J Exp Bio. Med Sci 1982, 60:175-180.

4. Yeo TW, Lampah DA, Gitawati R, Tjitra E, Kenangalem E, McNeil YR, Darcy CJ, Granger DL, Weinberg JB, Lopansri BK, Price RN, Duffull SB, Celermajer DS, Anstey NM: Impaired nitric oxide bioavailability and L-arginine reversible endothelial dysfunction in adults with falciparum malaria. J Exp Med 2007, 204:2693-2704.

5. Olszewski KL, Morrisey JM, Wilinski D, Burns JM, Vaidya AB, Rabinowitz JD, Llinás M: Host-parasite interactions revealed by Plasmodium falciparum metabolomics. Cell Host Microbe 2009, 19:191-199.

6. Gardner MJ, Hall N, Fung E, White OR, Berriman M, Hyman RW, Carlton JM-R, Pain A, Nelson KE, Bowman S, Paulsen IT, James K, Eisen JA, Rutherford KM, Salzberg SL, Craig AG, Kyes SA, Chan M-S, Nene V, Shallom SJ, Suh BB, Peterson JD, Angiuoli SV, Pertea M, Allen JE, Selengut JD, Haft D, Mather MW, Vaidya AB, Martin DM, Fairlamb AH, Fraunholz MJ, Roos DS, Ralph SA, McFadden Gl, Cummings LM, Subramanian GM, Mungall C, Venter JC, Carucci DJ, Hoffman SL, Newbold C, Davis RW, Fraser CM, Barrell B: Genome sequence of the human malaria parasite Plasmodium falciparum. Nature 2002, 419:498-511. 
7. Krugliak M, Zhang J, Ginsburg H: Intraerythrocytic Plasmodium falciparum utilizes only a fraction of the amino acids derived from the digestion of host cell cytosol for the biosynthesis of its proteins. Mol Biochem Parasitol 2002, 119:249-256

8. Siddiqui WA, Schnell JV: In vitro and in vivo studies with Plasmodium falciparum and plasmodium knowlesi. Proc Helm Soc Wash 1972, 39:204-210.

9. Divo AA, Geary TG, Davis NL, Jensen JB: Nutritional requirements of Plasmodium falciparum in culture, I: exogenously supplied dialyzable components necessary for continuous growth. J Protozool 1985, 32:59-64.

10. Bertagna P, Cohen S, Geiman QM, Haworth J, Koenigk E, Richards WH, Trigg PI: Cultivation techniques for the erythrocytic stages of malaria parasites. Bull World Health Organ 1972, 47:357-373.

11. Liu J, Istvan ES, Gluzman IY, Gross J, Goldberg DE: Plasmodium falciparum ensures its amino acid supply with multiple acquisition pathways and redundant proteolytic enzyme systems. Proc Natl Acad Sci USA 2006, 103:8840-8845.

12. Hill RJ, Konigsberg W, Guidotti G, Craig LC: The structure of human hemoglobin. I: the separation of the alpha and beta chains and their amino acid composition. J Biol Chem 1962, 237:1549-1554.

13. Watkins SM, German JB: Toward the implementation of metabolomic assessments of human health and nutrition. Curr Opin Biotechnol 2002, 13:512-516.

14. Noguchi Y, Zhang QW, Sugimoto T, Furuhata Y, Sakai R, Mori M, Takahashi $M$, Kimura T: Network analysis of plasma and tissue amino acids and the generation of an amino index for potential diagnostic use. Am J Clin Nutr 2006, 83:513S-519S.

15. Noguchi Y, Shikata N, Furuhata Y, Kimura T, Takahashi M: Characterization of dietary protein-dependent amino acid metabolism by linking free amino acids with transcriptional profiles through analysis of correlation Physiol Genomics 2008, 34:315-326.

16. Miyagi $Y$, Higashiyama M, Gochi A, Akaike M, Ishikawa T, Miura T, Saruki N, Bando E, Kimura H, Imamura F, Moriyama M, Ikeda I, Chiba A, Oshita F, Imaizumi A, Yamamoto H, Miyano H, Horimoto K, Tochikubo O, Mitsushima $\mathrm{T}$, Yamakado M, Okamoto N: Plasma free amino acid profiling of five types of cancer patients and its application for early detection. PLoS One 2011, 6:49-53.

17. Kell DB: Metabolomics and systems biology: making sense of the soup. Curr Opinion Microbiol 2004, 7:296-307.

18. Bannister $\mathrm{LH}$ : The interactions of intracellular protista and their host cells, with special reference to heterotrophic organisms. Proc $R$ Soc London, Ser B 1979, 204:141-163.

19. Herbas MS, Ueta YY, Ichikawa C, Chiba M, Ishibashi K, Shichiri M, Fukumoto S, Yokoyama N, Takeya M, Xuan X, Arai H, Suzuki H: Alpha-tocopherol transfer protein disruption confers resistance to malarial infection in mice. Malar J 2010, 9:101.

20. Zheng W, Wang QH, Liu YJ, Liu J, Feng H, Wu JJ, Cao YM: Distinct hostrelated dendritic cell responses during the early stage of Plasmodium yoelii infection in susceptible and resistant mice. Parasite Immunol 2010, 32:324-334.

21. Hsu JM, Anthony WL, Rider AA: Free amino acids in plasma and tissue of rats born to underfed dams. Proc Soc Exp Biol Med 1975, 148:1087-1089.

22. Rojkind M, Kershenobich D: Hepatic fibrosis. Prog Liver Dis 1976, 5:294-310.

23. The R project for statistical computing; www.r-project.org

24. Noguchi $Y$, Sakai R, Kimura T: Metabolomics and its potential for assessment of adequacy and safety of amino acid intake. J Nutr 2003, 133:2097S-2100S.

25. Daily JP, Scanfeld D, Pochet N, Le Roch K, Plouffe D, Kamal M, Sarr O, Mboup S, Ndir O, Wypij D, Levasseur K, Thomas E, Tamayo P, Dong C, Zhou Y, Lander ES, Ndiaye D, Wirth D, Winzeler EA, Mesirov JP, Regev A: Distinct physiological states of Plasmodium falciparum in malaria-infected patients. Nature 2007, 450:1091-1095.

26. Lee JS, Heo J, Libbrecht L, Chu IS, Kaposi-Novak P, Calvisi DF, Mikaelyan A, Roberts LR, Demetris AJ, Sun Z, Nevens F, Roskams T, Thorgeirsson SS: A novel prognostic subtype of human hepatocellular carcinoma derived from hepatic progenitor cells. Nat Med 2006, 12:410-416.

27. Rajara S, Oono Y: NeatMap-non-clustering heat map alternatives in R. BMC Bioinforma 2010, 11:45.

28. Holmes E, Wilson ID, Nicholson JK: Metabolic phenotyping in health and disease. Cell 2008, 134:714-717.
29. Russo I, Babbitt S, Muralidharan V, Butle T, Oksma A, Goldberg DE: Plasmepsin V licenses Plasmodium proteins for export into the host erythrocyte. Nature 2010, 463:632-636.

30. Baumeister S, Winterberg M, Przyborski JM, Lingelbach K: The malaria parasite Plasmodium falciparum: cell biological peculiarities and nutritional consequences. Protoplasma 2010, 240:3-12

31. Kimura T, Noguchi Y, Shikata N, Takahashi M: Plasma amino acid analysis for diagnosis and amino acid-based metabolic networks. Curr Opin Clin Nutr Metab Care 2009, 12:49-53.

\section{doi:10.1186/1475-2875-12-19}

Cite this article as: Saiki et al:: Multivariable analysis of host amino acids in plasma and liver during infection of malaria parasite Plasmodium yoelii. Malaria Journal 2013 12:19.

\section{Submit your next manuscript to BioMed Central and take full advantage of:}

- Convenient online submission

- Thorough peer review

- No space constraints or color figure charges

- Immediate publication on acceptance

- Inclusion in PubMed, CAS, Scopus and Google Scholar

- Research which is freely available for redistribution

Submit your manuscript at www.biomedcentral.com/submit
C BioMed Central 